\title{
THE GEOMETRIC MINIMAL MODELS OF ANALYTIC SPACES
}

\author{
SHIHOKO ISHII AND PIERRE MILMAN
}

\begin{abstract}
This paper shows that an analytic space $X$ has a unique maximal model through which every proper surjective morphism from a non-singular analytic space to $X$ factors. This is called the geometric minimal model of $X$ and characterized by the contraction property of rational curves. Some other properties such as functoriality, the direct product property and the quotient property of the geometric minimal model are also studied here. The relation of the geometric minimal model with Mori's minimal model is discussed.
\end{abstract}

\section{INTRODUCTION}

It is well known that a two dimensional singularity $(X, x)$ has a good birational model called the minimal resolution $f: Y \rightarrow X$, that is :

(1) all resolutions of $(X, x)$ factor through $f$ and

(2) $Y$ itself is non-singular.

For the higher dimensional case, such a resolution no longer exists. Then, seeking a good birational model of a higher dimensional singularity in the Minimal Model Conjecture, people modify the conditions (1) and (2) into a condition (3) which is equivalent to (1) and (2) in the two-dimensional case.

(3) $Y$ has $\mathbb{Q}$-factorial terminal singularities and $K_{Y}$ is $f$-nef.

As is known, a model satisfying (3) exists for a three-dimensional singularity ([12]), but the existence for the higher dimensional case is still a conjecture.

On the other hand, the minimal resolution $f: Y \rightarrow X$ of a twodimensional singularity is also characterized as follows:

(1) all resolutions of $(X, x)$ factor through $f$ and

$\left(2^{\prime}\right) f: Y \rightarrow X$ is maximal among those satisfying (1).

This paper shows that such a model also uniquely exists for the higher dimensional case.

We also prove that a model $f: Y \rightarrow X$ satisfying the following conditions $\left(1^{\prime}\right),\left(2^{\prime \prime}\right)$ uniquely exists for any dimension: 
$\left(1^{\prime}\right)$ all proper surjective morphisms $\tilde{X} \rightarrow X$ from non-singular $\tilde{X}$ factor through $f$ and

$\left(2^{\prime \prime}\right) f: Y \rightarrow X$ is maximal among those satisfying $\left(1^{\prime}\right)$.

This latter model is called the geometric minimal model of $X$ and is proved to coincide with the model satisfying (1) and $\left(2^{\prime}\right)$ if $\operatorname{dim} X \neq 2$.

The geometric minimal model is characterized by the contraction of rational curves: let $\tilde{X} \rightarrow X$ be a proper surjective morphism from non-singular $\tilde{X}$, then every rational curve on $\tilde{X}$ which is mapped to a point on $\mathrm{X}$ is mapped to a point on the geometric minimal model $Y$; conversely, the maximal model $Y \rightarrow X$ satisfying the following property coincides with the geometric minimal model: there is a proper bimeromorphic morphism $Z \rightarrow Y$ such that every rational curve on $Z$ which is mapped to a point on $X$ is mapped to a point on $Y$. By this, we can see that the geometric minimal model of a toric variety is the identity and the geometric minimal model of a cone over an Abelian variety is the corresponding line bundle over the Abelian variety.

In $\S 2$, we prove the existence of a maximal model through which all resolutions factor. The proof is done under a more general setting, and the existence of the geometric minimal model is also proved.

In $\S 3$, we prove the characterization of the geometric minimal model by the contraction of rational curves. The coincidence of the geometric minimal model and the model satisfying $(1)$ and $\left(2^{\prime}\right)$ for $\operatorname{dim} \neq 2$ is also proved here.

In $\S 4$, we prove miscellaneous properties of the geometric minimal model: functoriality, liftability of group actions, the quotient property, the direct product property; we also relate it with Mori's minimal model in the 3-dimensional case.

Throughout this paper, an analytic space $X$ is an analytic $\mathbb{C}$-space in Hironaka's sense [9], i.e.,

(i) for every point $x$ of $X$, there exists an open neighbourhood such that the restriction of $X$ onto it is $\mathbb{C}$-isomorphic to a local analytic $\mathbb{C}$-space,

(ii) the underlying topological space of $X$ is countable at infinity, i.e., $X$ is a union of countably many compact subsets, and

(iii) $X$ is a Hausdorff space.

Analytic spaces are always assumed to be irreducible and reduced except for Lemma 2.4.

Professor Hajime Tsuji pointed out that the technique used to prove Theorem 3.4 in the preliminary version is the same as that used in Fujiki's paper [8], thus our original proof could be shortened by quoting [8], for which the authors are grateful. The authors also would like to thank Professors Kenji Matsuki, Hironobu Maeda, Noboru Nakayama 
and David Mathieu for providing useful information. The authors express their heartfelt thanks to the referee for the numerous constructive comments.

\section{The greatest COMMON FACTOR OF A CATEgory OF PROPER SURJECTIVE MORPHISMS}

Definition 2.1. For an analytic space $X$, denote by $\mathcal{P S}(X)$ the category whose objects are proper surjective morphisms $X^{\prime} \rightarrow X$ from analytic spaces $X^{\prime}$ to $X$ and whose morphisms are proper surjective morphisms over $X$.

In this category, we introduce an order between the objects according to the existence of morphisms: i.e., $\left(X^{\prime} \rightarrow X\right) \geq\left(X^{\prime \prime} \rightarrow X\right)$, if there is a proper surjective morphism $X^{\prime} \rightarrow X^{\prime \prime}$ over $X$. In this case we say that $\left(X^{\prime} \rightarrow X\right)$ is greater than $\left(X^{\prime \prime} \rightarrow X\right)$. (Here note that "greater than" contains the case "equal to.")

Definition 2.2. For a subcategory $\mathcal{F}$ of $\mathcal{P} \mathcal{S}(X)$, an object $f: Y \rightarrow X$ of $\mathcal{P} \mathcal{S}(X)$ is called a common factor of $\mathcal{F}$, if every object of $\mathcal{F}$ is greater than $f$.

A maximal object among the common factors of $\mathcal{F}$ is called a maximal common factor of $\mathcal{F}$, and if a maximal common factor is unique, it is called the greatest common factor. In a slight abuse of terminology, we sometimes say that the space $Y$ is a common factor or the greatest common factor instead of referring to the morphism $Y \rightarrow X$.

For an object $\tilde{X} \rightarrow X$ and a common factor $Y \rightarrow X$ of $\mathcal{F}$, there is a morphism $\tilde{X} \rightarrow Y$ over $X$ by definition. We call this morphism a factorizing morphism. If $Y \rightarrow X$ is bimeromorphic, then a factorizing morphism is unique.

Note that if $\mathcal{F}^{\prime}$ is a subcategory of $\mathcal{F}$, a common factor of $\mathcal{F}$ is a common factor of $\mathcal{F}^{\prime}$. In particular the greatest common factor of $\mathcal{F}^{\prime}$ is greater than the greatest common factor of $\mathcal{F}$, if these greatest common factors exist.

Theorem 2.3. Assume that in a subcategory $\mathcal{F}$ of $\mathcal{P} \mathcal{S}(X)$ there is an object $X^{\prime} \rightarrow X$ which is bimeromorphic.

Then, for every common factor $Y^{\prime} \rightarrow X$ of $\mathcal{F}$ there is a greatest common factor $Y \rightarrow X$ such that $(Y \rightarrow X) \geq\left(Y^{\prime} \rightarrow X\right)$.

If for every object $X^{\prime} \rightarrow X$ of $\mathcal{F}, X^{\prime}$ is normal, then the greatest common factor of $\mathcal{F}$ is normal.

To prove the theorem, we need the following lemma and definition. 
Lemma 2.4. Let $\cdots E_{i+1} \stackrel{\varphi_{i+1}}{\longrightarrow} E_{i} \stackrel{\varphi_{i}}{\longrightarrow} E_{i-1} \cdots \rightarrow E_{0}$ be a sequence of proper surjective morphisms of reduced (not necessarily irreducible) analytic spaces. For each $i \geq 0$, let $\psi_{i}: E \rightarrow E_{i}$ be a proper surjective morphism of reduced analytic spaces. Assume the commutativity $\psi_{i}=$ $\varphi_{i+1} \circ \psi_{i+1}$ for every $i \geq 0$.

Then, for every compact subset $K \subset E_{0}$, there exists a number $r \geq 0$ such that $\varphi_{i}$ restricted onto the inverse image of $K$ is of finite fibers for all $i \geq r$.

Proof. Since we restrict onto a compact subset, we may assume that the number of irreducible components of $E$ is finite. Then, we may assume that the number of irreducible components of $E_{i}$ 's is constant, say $s$, because they are bounded by that of $E$. Let $E_{i}^{j}(j=1, . ., s)$ be the irreducible components of $E_{i}$ and $\varphi_{i+1}\left(E_{i+1}^{j}\right)=E_{i}^{j}$. Note that for every $j$ there is an irreducible component $E^{j}$ of $E$ which is mapped onto $E_{i}^{j}$. Then, for each $j$, we may assume that $\operatorname{dim} E_{i}^{j}$ 's are constant for all $i$, because they are bounded by $\operatorname{dim} E$. Now let us prove the lemma by induction on $n=\operatorname{dim} E$.

Assume $n=1$. Then $\operatorname{dim} E_{i}{ }^{j}=0$ for all $i$ or $\operatorname{dim} E_{i}^{j}=1$ for all $i$. Both cases are trivial.

Let $n \geq 2$ and assume that the lemma holds true whenever $\operatorname{dim} E<$ $n$. Since it is sufficient to prove the finiteness of $\varphi_{i}$ on each irreducible component $E_{i}^{j}$, we may assume that $E_{i}$ 's and $E$ are all irreducible. Let $\operatorname{dim} E_{i}=k$ and $\Phi_{i}=\varphi_{1} \cdots \varphi_{i-1} \circ \varphi_{i}$. Define $D_{i} \subset E_{i}$ as $\left\{x \in E_{i} \mid\right.$ $\left.\operatorname{dim}_{x} \Phi_{i}^{-1} \circ \Phi_{i}(x)>0\right\}$, then it is a proper closed subset of $E_{i}$. Next define $D \subset E$ as $\left\{y \in E \mid \operatorname{dim}_{y} \psi_{0}^{-1} \circ \psi_{0}(y)>n-k\right\}$, then it is also a proper closed subset in $E$ and satisfies $D_{i} \subset \psi_{i}(D)$. This inclusion is proved as follows:

For any point $x \in D_{i}$, take an irreducible component $D_{i 0}$ of $\Phi_{i}^{-1}$ 。 $\Phi_{i}(x)$ containing $x$, then $\operatorname{dim} D_{i 0}>0$. Let $D^{\prime} \subset E$ be an irreducible component of $\psi_{i}^{-1}\left(D_{i 0}\right)$ that dominates $D_{i 0}$ and $y \in D^{\prime}$ a point corresponding to $x$. Then, according to a general theory (see, for example, [13, Chap.1, §8, Theorem 2]), $\operatorname{dim} D^{\prime} \geq \operatorname{dim} D_{i 0}+n-k$. By $D^{\prime} \subset \psi_{0}^{-1} \circ \psi_{0}(y)$, we obtain that $\operatorname{dim}_{y} \psi_{0}^{-1} \circ \psi_{0}(y)>n-k$, which yields $x \in \psi_{i}(D)$.

Now we obtain a sequence $\cdots \psi_{i}(D) \rightarrow \psi_{i-1}(D) \rightarrow \cdots \rightarrow \psi_{0}(D)$ of proper surjective morphisms of reduced schemes which are dominated by $D$ of dimension $<n$. By the induction hypothesis, $\psi_{i}(D) \rightarrow \psi_{i-1}(D)$ is of finite fibers for $i>>0$. On the other hand $\varphi_{i}: E_{i} \rightarrow E_{i-1}$ is of finite fibers outside of $D_{i}$, therefore $\varphi_{i}$ is of finite fibers on the whole of $E_{i}$ for $i>>0$. 
Definition 2.5. Let $K$ be a compact subset of $X$. We say that a common factor $Y \rightarrow X$ is maximal on $K$, if for every common factor $Z \rightarrow X$ such that $(Z \rightarrow X) \geq(Y \rightarrow X)$ we have $\left.Z\right|_{K}=\left.Y\right|_{K}$, where $\left.\right|_{K}$ means the inverse image of $K$.

Proof of Theorem 2.3. First note that all common factors of $\mathcal{F}$ are bimeromorphic to $X$ by the assumption of the theorem. Let $Y \rightarrow X$ be a common factor of $\mathcal{F}$. We will prove that for a compact subset $K$, there exists a common factor $Y^{\prime} \rightarrow X$ such that $\left(Y^{\prime} \rightarrow X\right) \geq(Y \rightarrow X)$ and maximal on $K$. If $Y$ is not maximal on $K$, there is a common factor $Y_{1} \rightarrow X$ greater than $Y=Y_{0}$ and not equal to $Y_{0} \rightarrow X$ on $K$. Next, if $Y_{1}$ is not maximal on $K$, there is $Y_{2} \rightarrow X$ as above. Successively we obtain a sequence:

$$
\cdots \rightarrow Y_{i} \stackrel{\varphi_{i}}{\longrightarrow} Y_{i-1} \rightarrow \cdots \stackrel{\varphi_{1}}{\longrightarrow} Y_{0}
$$

of common factors of $\mathcal{F}$. Let $X^{\prime} \rightarrow X$ be an object of $\mathcal{F}$ and $\psi_{i}: X^{\prime} \rightarrow$ $Y_{i}$ the factorizing morphism for every $i$. Since $Y_{i}$ 's are bimeromorphic to $X$, the commutativity $\varphi_{i} \circ \psi_{i}=\psi_{i-1}$ follows for every $i$. Take the normalizations $X^{\prime *}$ and $Y_{i}^{*}$ of $X^{\prime}$ and $Y_{i}$, respectively. Then by the lemma, there is an integer $r=r(K)$ such that for every $i>r$ we have that $Y_{i+1}^{*} \rightarrow Y_{i}^{*}$ is a proper bimeromorphic morphism between normal spaces, and its restriction onto the inverse images of $K$ is of finite fibers, which is therefore an isomorphism by the Zariski Main Theorem (for an analytic version, see [6, 4.9]). Hence, on $K$, every common factor in

$$
\cdots \rightarrow Y_{i} \stackrel{\varphi_{i}}{\longrightarrow} Y_{i-1} \rightarrow \cdots \stackrel{\varphi_{r+1}}{\longrightarrow} Y_{r}
$$

is dominated by the common normalization $\pi_{i}: Y^{*} \rightarrow Y_{i}$. Therefore, the coherent $\mathcal{O}_{Y_{r}}$-module $\pi_{r *} \mathcal{O}_{Y^{*}}$ has an ascending chain of submodules:

$$
\mathcal{O}_{Y_{r}} \subset \rho_{r+1 *} \mathcal{O}_{Y_{r+1}} \subset \ldots \subset \rho_{i_{*}} \mathcal{O}_{Y_{i}} \subset \ldots
$$

where $\rho_{i}: Y_{i} \rightarrow Y_{r}$ is the composite $\varphi_{r} \circ \cdots \circ \varphi_{i}$. By the coherence of $\pi_{r *} \mathcal{O}_{Y^{*}}$ this chain should terminate at a finite stage. Thus, we obtain a common factor $Y^{\prime} \rightarrow X$ greater than $Y$ and maximal on $K$.

Since $X$ is countable at infinity, $X=\bigcup_{i=1}^{\infty} K_{i}$ with $K_{i}$ compact and $K_{i} \subset K_{i+1}$. Take a sequence

$$
\cdots \rightarrow Y_{i}^{\prime} \stackrel{\varphi_{i}^{\prime}}{\longrightarrow} Y_{i-1}^{\prime} \rightarrow \cdots \stackrel{\varphi_{1}^{\prime}}{\longrightarrow} Y_{0}=Y
$$

of common factors such that $Y_{i}^{\prime} \rightarrow X$ is maximal on $K_{i}$. Then,

$$
\cdots \rightarrow Y_{i}^{\prime} \stackrel{\varphi_{i}^{\prime}}{\longrightarrow} Y_{i-1}^{\prime} \rightarrow \cdots \stackrel{\varphi_{n+1}^{\prime}}{\longrightarrow} Y_{n}^{\prime}
$$

are all isomorphic on $K_{n}$. 
Now, as in [10, 4.1.7], we can define an analytic space $Y_{\infty}=\underset{\leftarrow}{\lim } Y_{r}^{\prime}$, which is again a common factor of $\mathcal{F}$ and greater than all $Y_{i}^{\prime}$ 's. This $Y_{\infty}$ is a maximal common factor of $\mathcal{F}$. Indeed, if $Z \rightarrow X$ is a common factor greater than $Y_{\infty}$, then for every $K_{i},\left.Z\right|_{K_{i}}=\left.Y_{i}^{\prime}\right|_{K_{i}}=\left.Y_{\infty}\right|_{K_{i}}$ by the maximality of $Y_{i}^{\prime}$ on $K_{i}$. Hence $Z=Y_{\infty}$.

Let $Y \rightarrow X$ and $Y^{\prime} \rightarrow X$ be maximal common factors. Then the unique component $Z$ of $Y \times_{X} Y^{\prime}$ dominating $Y$ and $Y^{\prime}$ is again a common factor of $\mathcal{F}$ and greater than $Y$ and $Y^{\prime}$, because of the universality of the fiber product. By the maximality of $Y$ and $Y^{\prime}, Z$ coincides with $Y$ and $Y^{\prime}$, which yields the uniqueness of the greatest common factor.

If every object of $\mathcal{F}$ is normal, the normalization of a common factor is also a common factor. So the greatest common factor is normal.

Remark 2.6. Instead of Lemma 2.4, we can prove the theorem differently: Consider a sequence of common factors as in the proof of Theorem 2.3. Each $Y_{i}$ is dominated by a common object $\tilde{X} \stackrel{f_{i}}{\longrightarrow} Y_{i}$. Let $\mathcal{J}_{i, a}$ be the defining ideal of $f_{i}^{-1} f_{i}(a)$ in $\tilde{X}$. On a compact subset $K$ on $Y_{0}$, consider the Hilbert-Samuel function $H_{i, a}$ of $\mathcal{O}_{\tilde{X}, a} / \mathcal{J}_{i, a}$ for $a \in f_{0}^{-1}(K)$. One can prove that there exists $n(K)$ such that for any $n \geq n(K)$ and $a \in f_{0}^{-1}(K)$, the equality $H_{n, a}=H_{n(K), a}$ holds by means of Zariski upper-semicontinuity ([2, lemmas 7.1, 7.2], [5, Lemma 3.10 and Definition 3.11]) and the stabilization property of Hilbert-Samuel function ([3], Theorem 5.2.1]). This equality yields the equality of the ideals $\mathcal{J}_{n, a}=\mathcal{J}_{n(K), a}$, and therefore $Y_{n} \rightarrow Y_{n(K)}$ is finite on $K$. The rest of the proof follows in the same way as above.

Remark 2.7. Consider the case that $X$ is an algebraic variety. Theorem 2.3 holds also for the category $\mathcal{P} \mathcal{S}$ alg $(X)$ whose objects are proper surjective morphisms of algebraic varieties to $X$. The proof is easier. In Lemma 2.4, there is a number $r$ so that $\varphi_{i}(i \geq r)$ is of finite fibers for the global $E_{i}$ 's. Therefore one can prove Theorem 2.3 immediately by showing the ascending chain condition, without using $Y_{\infty}$.

Example 2.8. There are many subcategories of $\mathcal{P} \mathcal{S}(X)$ which satisfy the condition of the theorem.

$\mathcal{R} e s(X)$ : the category of resolutions of the singularities of $X$. Here a resolution $f: \tilde{X} \rightarrow X$ means that $f$ is a proper bimeromorphic morphism from non-singular analytic space $\tilde{X}$.

$\mathcal{S} m(X)$ : the category of proper surjective morphisms $X^{\prime} \rightarrow X$, where $X^{\prime}$ is non-singular. Here, we note that the dimension of $X^{\prime}$ may be bigger than the dimension of $X$. 
THE GEOMETRIC MINIMAL MODELS OF ANALYTIC SPACES

$\mathcal{C}(X)$ (resp. $\mathcal{L} t(X), \mathcal{T}(X))$ : the category of proper bimeromorphic morphisms $X^{\prime} \rightarrow X$ where $X^{\prime}$ has canonical singularities (resp. logterminal singularities, terminal singularities).

These are of course nonempty categories due to the results of [9], [1], [4], [5] and [15].

We can also think of the following categories for a toric variety $X$ (cf., 2.7):

$\mathcal{R} e s(X)_{\text {tor }}$ : the category of toric resolutions of the singularities of $X$.

$\mathcal{S} m(X)_{\text {tor }}$ : the category of toric proper surjective morphisms $X^{\prime} \rightarrow$ $X$, where $X^{\prime}$ is non-singular.

We do not know if the greatest common factors of $\mathcal{S} m(X)$ and of $\mathcal{S} m(X)_{\text {tor }}$ are different for the same $X$.

Example 2.9. For a two-dimenstional analytic space $X$, the greatest common factor of $\mathcal{R} e s(X)$ is the minimal resolution.

Definition 2.10. The greatest common factor of $\mathcal{S} m(X)$ is called the geometric minimal model of $X$.

Definition 2.11. For an algebraic variety $X$, the greatest common factor of $\mathcal{S} m(X)$ in the category $\mathcal{P} \mathcal{S} a l g(X)$ is called the algebraic geometric minimal model which is not studied in the following sections (cf. 2.7).

\section{The GeOMetric Minimal MOdel AND RAtional CURVES}

In this section we study some properties of the geometric minimal model of an analytic space $X$.

First, we state and prove a basic lemma, which is well known and used often in this paper.

Lemma 3.1. Let $f: Z \rightarrow X$ be a proper surjective morphism and $g: Z \rightarrow Y$ a morphism. Assume $X$ is normal and $g$ is constant on $f^{-1}(x)$ for every $x \in X$.

Then, there is a morphism $\varphi: X \rightarrow Y$ such that $g=\varphi \circ f$.

Proof. Let $\Gamma \subset X \times Y$ be the reduced image of the proper morphism $(f, g): Z \rightarrow X \times Y$ and $\Phi: \Gamma \rightarrow X$ the restriction of the projection $X \times Y \rightarrow X$ onto $\Gamma$. Then, $\Phi^{-1}(x)$ consists of one point. Now we obtain that $\Phi: \Gamma \rightarrow X$ is a bijective proper bimeromorphic morphism. Since $X$ is normal, $\Phi$ is an isomorphism by Zariski's Main Theorem.

The following is a basic property of the geometric minimal model. 
Theorem 3.2. Let $X$ be an analytic space of dimension $\geq 1, \varphi: Y \rightarrow$ $X$ a common factor of $\mathcal{S} m(X)$ and $f: X^{\prime} \rightarrow X$ an arbitrary object of $\mathcal{S} m(X)$.

Then every rational curve $l \subset X^{\prime}$ which is mapped to a point on $X$ is mapped to a point on $Y$ by the factorizing morphism $X^{\prime} \rightarrow Y$.

Proof. It is sufficient to construct a commutative diagram with $f, \gamma$ and $f \circ \alpha=\gamma \circ \beta$ being in $\operatorname{Sm}(X)$

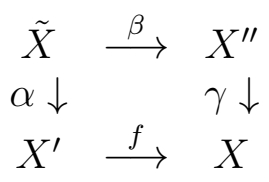

such that there is a curve $\tilde{l}$ on $\tilde{X}$ with $\alpha(\tilde{l})=l$ and $\beta(\tilde{l})=p$ a point. This is because $\gamma$ and $f$ factor through $\varphi: Y \rightarrow X$, and the image of $l$ on $Y$ coincides with the image of $\tilde{l}$ and therefore with the image of $p$.

Now we construct $\tilde{X}$ and $\tilde{l}$. First, if $\operatorname{dim} X^{\prime}=1$, then there is no such rational curve and therefore the statement is trivial. If $\operatorname{dim} X^{\prime}=2$, take the product $X_{0}=X^{\prime} \times \mathbb{P}^{1}$. Then, the composite $X_{0}=X^{\prime} \times \mathbb{P}^{1} \stackrel{p_{1}}{\longrightarrow}$ $X^{\prime} \rightarrow X$ is also an object of $\mathcal{S} m(X)$, where $p_{1}$ is the projection to the first factor. Take a section $l_{0}$ of $p_{1}$ over $l$. If $\operatorname{dim} X^{\prime} \geq 3$, let $X_{0}=X^{\prime}$ and $l_{0}=l$.

Then for $\operatorname{dim} X^{\prime} \geq 2$, there is a proper bimeromorphic morphism $\alpha_{1}: X_{1} \rightarrow X_{0}$ with a non-singular rational curve $l_{1} \subset X_{1}$ such that $\alpha_{1}\left(l_{1}\right)=l_{0}$ and the normal sheaf $N_{l_{1} / X_{1}}=\mathcal{O}_{\mathbb{P}^{1}}(-1) \oplus \mathcal{O}_{\mathbb{P}^{1}}(-1) \oplus \cdots \oplus$ $\mathcal{O}_{\mathbb{P}^{1}}(-1)$ by Fujiki's result $[8$, Lemma 5]. The following construction is also found in [8]: take the blow-up $\alpha_{2}: \tilde{X} \rightarrow X_{1}$ with the center $l_{1}$ and define $\alpha:=\alpha_{1} \circ \alpha_{2}$. Then the exceptional divisor $E$ of $\alpha_{2}$ is $\mathbb{P}^{1} \times \mathbb{P}^{n-2}$ and its normal sheaf is $p_{1}^{*} \mathcal{O}_{\mathbb{P}^{1}}(-1) \otimes p_{2}^{*} \mathcal{O}_{\mathbb{P}^{n-2}}(-1)$, where $p_{i}$ is the projection to the $i$-th factor and $n$ is the dimension of $X^{\prime}$. Then, there is a proper bimeromorphic morphism $\beta: \tilde{X} \rightarrow X^{\prime \prime}$ whose restriction onto $E$ is the projection $E \stackrel{p_{2}}{\longrightarrow} \mathbb{P}^{n-2}$. By the normal bundle of $E$, we can see that $X^{\prime \prime}$ is non-singular (see, for example, [11, Theorem $3,(2)])$. Since $f \circ \alpha$ is constant on $\beta^{-1}(x)$ for every $x \in X^{\prime \prime}$, we obtain a morphism $\gamma: X^{\prime \prime} \rightarrow X$ with $\gamma \circ \beta=f \circ \alpha$ by Lemma 3.1. Now, take a section $\tilde{l}=\mathbb{P}^{1} \times\{P\} \subset E$ over $l_{1}$, where $P$ is a point of $\mathbb{P}^{n-2}$. Then $\beta(\tilde{l})$ is a point, which shows that $\tilde{l}$ is a curve we wanted.

Theorem 3.3. Let $X$ be an analytic space of dimension $\geq 3, \varphi^{\prime}$ : $Y^{\prime} \rightarrow X$ a common factor of $\mathcal{R} e s(X)$ and $f: X^{\prime} \rightarrow X$ an arbitrary object of $\operatorname{Res}(X)$. 
Then every rational curve $l \subset X^{\prime}$ which is mapped to a point on $X$ is mapped to a point on $Y^{\prime}$ by the factorizing morphism $X^{\prime} \rightarrow Y^{\prime}$.

Proof. Since all objects in $\mathcal{R} e s(X)$ are of dimension $\geq 3$, we can construct a commutative diagram in $\mathcal{R} e s(X)$ in the same way as in the proof of the previous theorem.

Theorem 3.4. Let $Y \rightarrow X$ be a bimeromorphic object of $\mathcal{P} \mathcal{S}(X)$ satisfying the following condition $(R)$ :

(R) There exists a proper bimeromorphic morphism $\tilde{Y} \rightarrow Y$ such that every rational curve $l \subset \tilde{Y}$ which is mapped to a point in $X$ is mapped to a point in $Y$.

Then $Y \rightarrow X$ is a common factor of $\operatorname{Sm}(X)$.

Proof. Let $X^{\prime} \rightarrow X$ be an arbitrary object of $\mathcal{S} m(X)$. Then, there is a component $Z$ of $X^{\prime} \times_{X} \tilde{Y}$ on which $X^{\prime} \times_{X} \tilde{Y} \rightarrow X^{\prime}$ is bimeromorphic. Then, by [9, Main Theorem II'] and [10, Corollary 1], for every point $x \in X^{\prime}$ there are a relatively compact neighbourhood $U_{r},(r \geq 1)$ of $x$, a non-singular analytic space $U_{1}$, bimeromorphic morphisms $f: U_{1} \rightarrow U_{r}$ and $\left.U_{1} \rightarrow Z\right|_{U_{r}}$ such that $f$ is the composite $f_{r-1} \circ f_{r-2} \circ \cdots \circ f_{1}$ of blow-ups $f_{i}: U_{i} \rightarrow U_{i+1}$ with non-singular centers and the following diagram is commutative:

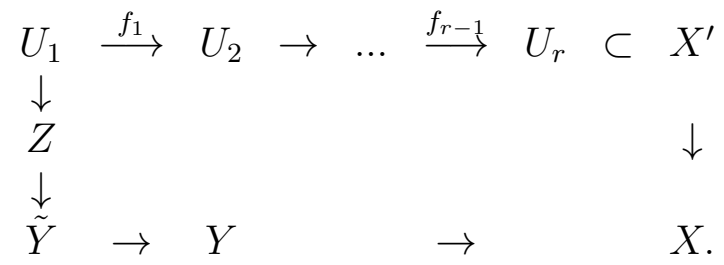

Therefore, $f_{1}^{-1}(y)=\mathbb{P}^{s}(s \geq 0)$ for every $y \in U_{2}$.

For a rational curve $l \subset f_{1}^{-1}(y) \subset U_{1}$, if the image of $l$ on $\tilde{Y}$ is not a point then it is a rational curve. So $l$ is mapped to a point in $Y$ by the condition (R), which yields the existence of a morphism $U_{2} \rightarrow Y$ by Lemma 3.1. In the same way we induce the morphisms $U_{i} \rightarrow Y$ for $i=2, . ., r$. Now we complete the proof of the statement that $X^{\prime} \rightarrow X$ factors through $Y \rightarrow X$.

Corollary 3.5. Let $X$ be an analytic space of dimension $\neq 2$.

Then, the greatest common factor of $\mathcal{R} e s(X)$ coincides with the geometric minimal model.

Proof. Since $\mathcal{R} e s(X) \subset \mathcal{S} m(X)$, it follows that the greatest common factor $Y^{\prime} \rightarrow X$ of $\mathcal{R} e s(X)$ is greater than the geometric minimal model $Y \rightarrow X$. For $\operatorname{dim} X=1, Y$ and $Y^{\prime}$ are normal and bimeromorphic to $X$, therefore they coincide by Theorem 2.3. 
For $\operatorname{dim} X \geq 3$, it is sufficient to prove that $Y^{\prime} \rightarrow X$ is a common factor of $\mathcal{S} m(X)$ by Theorem 2.3. This follows from Theorem 3.3 and Theorem 3.4.

Theorem 3.6. The geometric minimal model of an analytic space $X$ is the maximal bimeromorphic object among the objects satisfying $(R)$ in $\mathcal{P} \mathcal{S}(X)$.

Proof. This is clear by Theorem 2.3 and Theorem 3.4, because the geometric minimal model also satisfies $(R)$ by Theorem 3.2 .

Corollary 3.7. If $Y \rightarrow X$ is the geometric minimal model, then for every non-trivial bimeromorphic morphism $Z \rightarrow Y$, there is a rational curve on $Z$ which is mapped to a point on $Y$.

Proof. If $Y \rightarrow X$ is the geometric minimal model, then $Z$ is not a common factor of $\mathcal{S} m(X)$ by the maximality of $Y$. Therefore, by Theorem 3.4, for a resolution $\tilde{Z} \rightarrow Z$ of singularities of $Z$ there is a rational curve $l \subset \tilde{Z}$ which is mapped to a point on $X$ and to a curve $l^{\prime}$ on $Z$. This curve $l^{\prime}$ is a rational curve on $Z$, as required.

Now, the above theorem and Theorem 2.3 imply the following:

Corollary 3.8. If $X$ is an analytic space of dimension two, then the geometric minimal model of $X$ is a surface obtained by contracting all rational exceptional curves of the minimal resolution.

Example 3.9. Let $X$ be an affine cone over a non-singular variety $A$, which means that there is a line bundle $Y$ over $A$ with the zero section of negative normal bundle and the zero section is contracted to a point of $X$ in $Y$. We will denote the zero section of $Y$ also by $A$.

(1) Suppose $A$ is either an Abelian variety or the direct product of non-singular curves of positive genera. Then $Y$ is a resolution of the singularities on $X$ and also the geometric minimal model of $X$, since $A$ has no rational curve (see Theorem 3.4).

(2) If $A$ is an algebraic K3-surface, then there is a rational curve with positive self-intersection number. Then, the whole of $A$ should be contracted to a point in the geometric minimal model; therefore the geometric minimal model of $X$ is the identity.

(3) Let $A$ be a ruled surface with $q \geq 1$ and $p: A \rightarrow C$ the ruling, then there is a contraction $Y \rightarrow Y^{\prime}$ of the zero section $A$ to a curve $C$ in $Y$ by [7, Theorem 1]. Then $Y^{\prime}$ is the geometric minimal model of $X$. 
Example 3.10. Let $X \subset \mathbb{C}^{4}$ be a hypersurface defined by a homogeneous polynomial $f$ such that the hyperplane in $\mathbb{P}^{3}$ defined by $f$ is non-singular and hyperbolic (for the definition of hyperbolic, see $\S 4$ of 81). Then, the blow-up $\varphi: Y \rightarrow X$ of $X$ at the origin is the geometric minimal model of $X$, because there is no rational curve in a fiber of $\varphi$ and $Y$ is non-singular.

Example 3.11. Let $X$ be a toric variety, then the geometric minimal model is the identity. Indeed, for every toric resolution $f: X^{\prime} \rightarrow X$, each fiber $f^{-1}(x)$ of $f$ is a connected union of rational varieties, so it should be mapped to a point on the geometric minimal model.

\section{Miscellaneous properties on the GeOMetric Minimal MODELS}

Proposition 4.1. (Functoriality) Let $X$ and $X^{\prime}$ be analytic spaces and $\varphi: Y_{X} \rightarrow X$ and $\varphi^{\prime}: Y_{X^{\prime}} \rightarrow X^{\prime}$ the geometric minimal models of $X$ and $X^{\prime}$, respectively.

Then a proper surjective morphism $f: X \rightarrow X^{\prime}$ can be uniquely lifted to $\tilde{f}: Y_{X} \rightarrow Y_{X^{\prime}}$ such that $\varphi^{\prime} \circ \tilde{f}=f \circ \varphi$.

Proof. The composition with $f$ gives an injection $\mathcal{S} m(X) \rightarrow \mathcal{S} m\left(X^{\prime}\right)$. Therefore the greatest common factor of the image of $\mathcal{S} m(X)$ is greater than that of $\mathcal{S} m\left(X^{\prime}\right)$.

Corollary 4.2. Let $\pi: X \rightarrow S$ be a proper surjective morphism to a surface $S$. Let $S^{\prime}$ be a surface obtained by contracting all rational exceptional curves of the minimal resolution of $S$. If the geometric minimal model of $X$ is the identity (for example, $X$ is toric), then $\pi$ factors through $S^{\prime}$.

Corollary 4.3. Let $X$ be an analytic space and $\varphi: Y \rightarrow X$ the geometric minimal model of $X$.

Then every automorphism $\sigma: X \rightarrow X$ can be lifted to $\sigma^{\prime}: Y \rightarrow Y$ such that $\varphi \circ \sigma^{\prime}=\sigma \circ \varphi$.

Proof. By Proposition 4.1, $\sigma$ can be lifted to $\sigma^{\prime}: Y \rightarrow Y$ and $\sigma^{-1}$ can be lifted to $\sigma^{\prime \prime}: Y \rightarrow Y$. By the uniqueness of the lifting, $\sigma^{\prime}$ and $\sigma^{\prime \prime}$ are the inverse morphism of each other.

By this corollary, we obtain that every action of a finite group on $X$ is lifted to the geometric minimal model. What follows is a discussion of the quotient by a finite group. 
Proposition 4.4. Let $X$ be an analytic space and $G$ a finite group acting on $X$. Let $\varphi: Y_{X} \rightarrow X$ and $\varphi^{\prime}: Y_{X / G} \rightarrow X / G$ be the geometric minimal models of $X$ and $X / G$, respectively.

Then there is a morphism $Y_{X} / G \rightarrow Y_{X / G}$ over $X / G$.

Proof. By the functoriality of the geometric minimal models (cf. Proposition 4.1), there is a morphism $Y_{X} \rightarrow Y_{X / G}$. By the uniqueness of this morphism, it is a $G$-equivariant morphism with the trivial action on $Y_{X / G}$. Hence the morphism factors through $Y_{X} / G$.

The following is obvious from the above proposition.

Corollary 4.5. Let $X$ be an analytic space and $G$ a finite group acting on $X$. If the geometric minimal model of $X$ is the identity, then the geometric minimal model of $X / G$ is also the identity.

Example 4.6. Let $X=\mathbb{C}^{n} / G$ by a finite group $G$, then the geometric minimal model of $X$ is the identity.

Example 4.7. The equality $Y_{X} / G \simeq Y_{X / G}$ does not hold in general. Indeed, take a line bundle $Y^{\prime}=\mathbf{V}\left(\mathcal{O}_{\mathbb{P}^{1}}(m)\right)$ over $\mathbb{P}^{1}$, where $m$ is a positive integer. Let $\pi: C \rightarrow \mathbb{P}^{1}$ be a double cover from an elliptic curve $C$ and $G=\mathbb{Z} /(2)$ the Galois group. Then $G$ acts on $Y=\mathbf{V}\left(\pi^{*} \mathcal{O}_{\mathbb{P}^{1}}(m)\right)$. Denoting by $X$ the variety obtained by contracting the zero section of $Y$, the group $G$ acts on $X$ as well. The quotient $X / G$ has a resolution $Y^{\prime}$, therefore it is the quotient of $\mathbb{C}^{2}$ by a finite group of order $m$. Hence the geometric minimal model of $X / G$ is the identity, but the geometric minimal model of $X$ is $Y$, and $Y / G \simeq Y^{\prime}$.

In order to prove the direct product property of the geometric minimal model, we state the following lemma.

Lemma 4.8. Let $X$ and $X^{\prime}$ be analytic spaces, $\mathcal{F}$ a subcategory of $\mathcal{P S}(X)$ satisfying the condition of Theorem 2.3, i.e., there is a bimeromorphic object $X^{\prime} \rightarrow X$ in $\mathcal{F}$. Let $Y_{X}$ be the greatest common factor of $\mathcal{F}$ and $f: Z \rightarrow X^{\prime}$ an object of $\mathcal{P} \mathcal{S}\left(X^{\prime}\right)$. Let $\mathcal{F} \times Z$ be the category of the morphisms $\tilde{X} \times Z \rightarrow X \times X^{\prime}$ with $\tilde{X} \in \mathcal{F}$.

Then $Y_{X} \times Z$ is the greatest common factor of $\mathcal{F} \times Z$.

Proof. It is clear that $Y_{X} \times Z$ is a common factor of $\mathcal{F} \times Z$. By Theorem 2.3, there is the greatest common factor $Y^{\prime}$ of $\mathcal{F} \times Z$. So for every object $\tilde{X} \rightarrow X$ of $\mathcal{F}$, there is a factorizing morphism $\varphi: \tilde{X} \times Z \rightarrow Y^{\prime}$ of $\tilde{X} \times Z \rightarrow X \times X^{\prime}$. On the other hand, there is a morphism $\psi: Y^{\prime} \rightarrow$ $Y_{X} \times Z$ by the maximality of $Y^{\prime}$. Therefore for every point $z \in Z$, $\tilde{X} \times\{z\} \rightarrow X \times\{f(z)\}$ is factored as follows: 


$$
\tilde{X} \times\{z\} \stackrel{\varphi}{\longrightarrow} \psi^{-1}\left(Y_{X} \times\{z\}\right) \stackrel{\psi}{\longrightarrow} Y_{X} \times\{z\} \rightarrow X \times\{f(z)\} .
$$

So, $\psi^{-1}\left(Y_{X} \times\{z\}\right)$ is a common factor of $\mathcal{F}$. Then, by the maximality of $Y_{X}, \psi^{-1}\left(Y_{X} \times\{z\}\right) \stackrel{\psi}{\longrightarrow} Y_{X} \times\{z\}$ is an isomorphism. Now we obtain that $\psi: Y^{\prime} \rightarrow Y_{X} \times Z$ is bijective and $\psi^{-1}((y, z)) \simeq\{(y, z)\}$ for every point $(y, z) \in Y_{X} \times Z$, which yields that $\psi$ is an isomorphism.

Proposition 4.9. (Direct product) Let $X$ and $X^{\prime}$ be analytic spaces, $Y_{X}, Y_{X^{\prime}}$ and $Y_{X \times X^{\prime}}$ the geometric minimal models of $X, X^{\prime}$ and $X \times X^{\prime}$, respectively.

Then $Y_{X} \times Y_{X^{\prime}} \simeq Y_{X \times X^{\prime}}$ over $X \times X^{\prime}$.

Proof. First for any objects $\tilde{X} \rightarrow X$ and $\tilde{X}^{\prime} \rightarrow X^{\prime}$ of $\mathcal{S} m(X)$ and $\mathcal{S} m\left(X^{\prime}\right)$, respectively, $\tilde{X} \times \tilde{X}^{\prime} \rightarrow X \times X^{\prime}$ factors through $Y_{X \times X^{\prime}}$. For every $\tilde{X}^{\prime}$ in $\mathcal{S} m\left(X^{\prime}\right), Y_{X} \times \tilde{X}^{\prime}$ is the greatest common factor of $\mathcal{S} m(X) \times$ $\tilde{X}^{\prime}$ by Lemma 4.8. So there is a morphism $Y_{X} \times \tilde{X}^{\prime} \rightarrow Y_{X \times X^{\prime}}$. Then by Lemma 4.8 again, $Y_{X} \times Y_{X^{\prime}}$ is the maximal common factor of $Y_{X} \times$ $\mathcal{S} m\left(X^{\prime}\right)$. So there is a morphism $Y_{X} \times Y_{X^{\prime}} \rightarrow Y_{X \times X^{\prime}}$.

In order to prove the existence of the inverse morphism $Y_{X \times X^{\prime}} \rightarrow$ $Y_{X} \times Y_{X^{\prime}}$, it is sufficient to prove that $Y_{X} \times Y_{X^{\prime}}$ is a common factor of $\mathcal{S} m\left(X \times X^{\prime}\right)$. Let $\tilde{X}$ and $\tilde{X}^{\prime}$ be resolutions of $Y_{X}$ and $Y_{X^{\prime}}$, respectively, and $l$ a rational curve on $\tilde{X} \times \tilde{X}^{\prime}$ which is mapped to a point on $X \times X^{\prime}$. By Theorem 3.4, it is sufficient to prove that $l$ is mapped to a point on $Y_{X} \times Y_{X^{\prime}}$. If the image of $l$ by the projection $\tilde{X} \times \tilde{X}^{\prime} \stackrel{p_{1}}{\longrightarrow} \tilde{X}$ is not a point, then it is a rational curve, and therefore is mapped to a point on $Y_{X}$. In the same way, $l$ is mapped to a point on $Y_{X^{\prime}}$ through the projection $\tilde{X} \times \tilde{X}^{\prime} \stackrel{p_{2}}{\longrightarrow} \tilde{X}^{\prime}$. Hence $l$ is mapped to a point on $Y_{X} \times Y_{X^{\prime}}$.

Next we consider an action of a complex Lie group.

Proposition 4.10. Let $G$ be a complex Lie group acting on an analytic space $X$.

Then the action can be lifted to the geometric minimal model of $X$.

Proof. Let $\sigma: G \times X \rightarrow X$ be the morphism defining the action, $p_{1}: G \times X \rightarrow G$ the projection to the first factor and $Y$ the geometric minimal model of $X$. Since the geometric minimal model of $G \times X$ is $G \times Y$ by Proposition 4.9, the automorphism $\left(p_{1}, \sigma\right): G \times X \rightarrow G \times X$ can be lifted to $\Phi: G \times Y \stackrel{\sim}{\rightarrow} G \times Y$. The composite $p_{2} \circ \Phi: G \times Y \rightarrow Y$ defines the lifted action of $G$ on $Y$, where $p_{2}: G \times Y \rightarrow Y$ is the projection to the second factor. 
4.11. It is natural to ask if the geometric minimal model is a local property, i.e., if $\varphi: Y \rightarrow X$ is the geometric minimal model of $X$ and $U \subset X$ is open, then $\varphi^{-1}(U) \rightarrow U$ is the geometric minimal model of $U$. The following example shows that the geometric minimal model is not local.

Example 4.12. Here we construct $U \subset X$ such that the geometric minimal model of $X$ is the identity but that of $U$ is not.

Let $S$ be a compact rational surface with only one simple elliptic singularity $p$ on it. Let $V=S \times \mathbb{P}^{1}$ and $C \not \ngtr p$ be a non-singular very ample divisor on $S_{0}=S \times\{0\}$. Take the blow-up $X^{\prime} \rightarrow V$ with the center $C$, then the proper transform $S_{0}^{\prime}$ of $S_{0}$ on $X^{\prime}$ has the negative normal bundle on $X^{\prime}$. Hence, there is a morphism $f: X^{\prime} \rightarrow X$ which contracts $S_{0}^{\prime}$ to a point $Q$ of $X$. Since $X^{\prime}$ has singularities (simple elliptic singularities $) \times \mathbb{P}^{1}$, there is a resolution $g: \tilde{X} \rightarrow X^{\prime}$ whose exceptional divisor $E$ is isomorphic to (elliptic curve) $\times \mathbb{P}^{1}$. Then, the composite $f \circ g: \tilde{X} \rightarrow X$ factors through the geometric minimal model $Y \rightarrow X$ of $X$. The proper transform $S_{0}^{\prime \prime}$ of $S_{0}^{\prime}$ on $\tilde{X}$ is contracted to a point on $Y$, because $S_{0}^{\prime \prime}$ is rational. As $S_{0}^{\prime \prime} \cap E$ has the trivial normal bundle in $E$, the other elliptic fibers are all contracted to points on $Y$, therefore $Y$ must be isomorphic to $X$.

On the other hand, for $U=X \backslash\{Q\}$, the restriction $(f \circ g)^{-1}(U) \rightarrow U$ is a resolution of singularities on $U$ and has no rational curves in each fiber of $f \circ g$, therefore it is the geometric minimal model.

Proposition 4.13. Let $X$ be a three-dimensional analytic space with at worst canonical singularities.

Then the geometric minimal model of $X$ is the identity.

Proof. By [14, Corollary 2.14], every exceptional divisor of a resolution $f: \tilde{X} \rightarrow X$ is a ruled surface. Indeed, [14, Corollary 2.14] shows that there exists a resolution $X_{1}^{\prime} \rightarrow X^{\prime}$ of a canonical singularity $\left(X^{\prime}, x\right)$ of index one such that all exceptional divisors are ruled. For an arbitrary resolution $X_{2}^{\prime} \rightarrow X^{\prime}$, there exists a resolution $\tilde{X}^{\prime} \rightarrow X^{\prime}$ dominating both $X_{1}^{\prime}$ and $X_{2}^{\prime}$ such that the morphism $\tilde{X}^{\prime} \rightarrow X_{1}^{\prime}$ is the composite of blow-ups at non-singular centers. Therefore, all exceptional divisors for $\tilde{X}^{\prime} \rightarrow X^{\prime}$ are ruled which yields that all exceptional divisors for $X_{2}^{\prime} \rightarrow X^{\prime}$ are ruled. For a canonical singularity of index $>1$, take the canonical cover and reduce the problem to the case of index one. Here, we should note that a surface which is a surjective image of a ruled surface is again ruled.

As all exceptional divisors are ruled, the geometric minimal model $\varphi: Y \rightarrow X$ of $X$ is either an isomorphism or a contraction of the union 
$C$ of finitely many curves. Assume that $\varphi$ is not an isomorphism and let $g: \tilde{X} \rightarrow Y$ be the factorizing morphism. Then, all components of $C$ are rational, otherwise $R^{1} \varphi_{*} \mathcal{O}_{Y} \neq 0$, since there is a surjection $R^{1} \varphi_{*} \mathcal{O}_{Y} \rightarrow H^{1}\left(C_{i}, \mathcal{O}_{C_{i}}\right)$, where $C_{i}$ is a component of $C$. This nonvanishing contradicts the edge sequence

$$
0 \rightarrow E_{2}^{1,0} \rightarrow E^{1} \rightarrow \ldots
$$

of the Leray spectral sequence:

$$
E_{2}^{p, q}=R^{p} \varphi_{*} R^{q} g_{*} \mathcal{O}_{\tilde{X}} \Longrightarrow E^{p+q}=R^{p+q} f_{*} \mathcal{O}_{\tilde{X}} .
$$

If the curve $C$ is not contained in the singular locus of $Y$, then we can construct a resolution $\tilde{X}^{\prime} \rightarrow X$ with a rational curve $l$ on $\tilde{X}^{\prime}$ mapped to a point on $X$ and mapped to a curve on $Y$, a contradiction to Theorem 3.2. Thus, $C$ is contained in the singular locus of $Y$. Note that $Y$ has at worst canonical singularities, since $K_{Y}=\varphi^{*} K_{X}$ which follows from that $\varphi: Y \rightarrow X$ is isomorphic in codimension one. So general points of $C$ are cDV singularities in $Y$. For an irreducible component $C_{i}$ of $C$, take the blow-up $h: Y^{\prime} \rightarrow Y$ with the center $C_{i}$.

Then, in case $Y$ has compound $A_{1}, D_{n}(n \geq 4), E_{6}, E_{7}, E_{8}$ singularities on general points of $C_{i}, h$ has an irreducible exceptional divisor $E$ and $h^{-1}(p)$ is an irreducible rational curve for a general $p \in C_{i}$. This is because the restriction of $h$ onto a general hyperplane cut at a general point of $C_{i}$ is the blow-up of the surface at the point. Then, $E$ is rational and is not contained in the singular locus of $Y^{\prime}$. Therefore, by Theorem 3.2, $E$ must be mapped to a point on $Y$, a contradiction.

In case $Y$ has compound $A_{n}(n \geq 2)$ singularities at general points of $C_{i}$, then $h^{-1}(p)$ is the union of two $\mathbb{P}^{1}$ with one intersection point. This implies that the exceptional divisor $E$ of $h$ has a generic section over $C_{i}$. Then, take the blow-up $h_{2}: Y_{2} \rightarrow Y^{\prime}$ at the intersection curve of $E$ (this curve is the generic section over $C_{i}$ ). Then, again blow-up $Y_{2}$ at the intersection curve of the new exceptional divisor, if the intersection curve exists. Continuing this procedure, we obtain a sequence of $([n / 2]+1)$-blow-ups at generic sections over $C_{i}$ :

$$
Y_{[n / 2]+1} \stackrel{h_{[n / 2]+1}^{\longrightarrow}}{\longrightarrow} Y_{[n / 2]} \rightarrow \ldots \stackrel{h_{2}}{\longrightarrow} Y^{\prime} \stackrel{h}{\longrightarrow} Y .
$$

We can see that the exceptional divisor $E_{[n / 2]+1}$ of the last blow-up is irreducible and the fiber $h_{[n / 2]+1}^{-1} \circ \cdots \circ h^{-1}(p)$ on $E_{[n / 2]+1}$ of a general point $p \in C_{i}$ is $\mathbb{P}^{1}$. It follows that $E_{[n / 2]+1}$ is rational and is not contained in the singular locus of $Y_{[n / 2]+1}$, a contradiction in the same way as above. 
Corollary 4.14. Let $X$ be a sufficiently small neighbourhood of a logterminal singularity of dimension three.

Then the geometric minimal model of $X$ is the identity.

Proof. Taking the canonical cover, we may assume that $X$ is a cyclic quotient of an analytic space with at worst canonical singularities. Then, by Corollary 4.5 and Proposition 4.13 we obtain the statement.

Corollary 4.15. Let $X$ be an analytic space of dimension three, $Y^{\prime} \rightarrow$ $X$ the relative canonical model in Mori's sense and $Y \rightarrow X$ the geometric minimal model.

Then there is a morphism $\varphi: Y^{\prime} \rightarrow Y$ over $X$. Therefore, there is a morphism $Y^{\prime \prime} \rightarrow Y$ over $X$, where $Y^{\prime \prime}$ is a relative minimal model in Mori's sense.

Proof. By 1.1, the geometric minimal model of $Y^{\prime}$ is greater than $Y$ over $X$. Here, by Proposition 4.13, the geometric minimal model of $Y^{\prime}$ is the identity.

\section{REFERENCES}

[1] J. M. Aroca, H. Hironaka \& J. Vicente Desingularization Theorems, Mem. Math. del Inst. "Jorge Juan" 30, (1977) Madrid.

[2] E. Bierstone \& P. Milman, Relations among analytic functions I, II, Ann, Inst. Fourier (Grenoble) 37 (1), (1987) 187-239, 37 (2), (1987) 49-77.

[3] E. Bierstone \& P. Milman, Uniformization of analytic spaces, J. Amer. Math. Soc. 2 (1989) 801-836.

[4] E. Bierstone \& P. Milman, A simple constructive proof of canonical resolution of singularities, Effective methods in algebraic geometry, Progress in Math. 94, Birkhäuser (1991) 11-30.

[5] E. Bierstone \& P. Milman, Canonical desingularization in characteristic zero by blowing up the maximum strata of a local invariant, Invent. Math. 128, (1997), 207-302

[6] G. Fischer, Complex Analytic Geometry, Lecture Notes in Math., 538, Springer-Verlag (1976).

[7] A. Fujiki, On the blowing down of analytic spaces, Publ. RIMS, Kyoto Univ. 10, (1975) 473-507.

[8] A. Fujiki, On the minimal models of complex manifolds, Math. Ann. 253, (1980) 111-128.

[9] H. Hironaka, Resolution of singularities of an algebraic variety over a field of characteristic zero: I, II, Annals of Math.,79, (1964) 109-326.

[10] H. Hironaka, Flattening Theorem in complex-analytic geometry, Am. J. Math., 97, (1975) 503-547.

[11] S. Ishii, Some projective contraction theorems, manuscripta math. 22, (1977) 343-358. 
[12] S. Mori, Flip theorem and the existence of minimal models for 3-folds, J. Amer. Math. Soc. 1, (1988) 117-253.

[13] D. Mumford, Red Book of Varieties and Schemes, Lecture Note in Math. 1358, Springer-Verlag, (1988).

[14] M. Reid, Canonical 3-folds, Proc. Algebraic Geometry Angers, Sijthoff and Nordhoff (1979) 273-310.

[15] O. Villamayor, Patching local uniformizations, Ann. Sci. l'École Norm. Sup. (4) 25, (1992) 629-677.

Shihoko Ishii: Department of Mathematics, Tokyo Institute of Technology, OH-OKayama, Meguro, TOKYo, JAPAN

Pierre Milman: Department of Mathematics, University of Toronto, Toronto, Ontario, Canada 